\title{
A Hydroxypropyl Chitosan (HPCH) Based Medical Device Prevents Fungal Infections: Evidences from an In Vitro Human Nail Model
}

\author{
Anna Bulgheroni*, Linda Frisenda, Alessandro Subissi and Federico Mailland
}

Scientific Department, Polichem S.A., Lugano CH, Switzerland

\begin{abstract}
A long lasting, protective and film forming HPCH-based medical device was developed and tested in a novel in vitro human nail infection model. HPCH-treated and untreated human distal fingernail fragments were disposed on the culture surface of Trychophyton rubrum, T. mentagrophytes, Scopulariopsis brevicaulis or Candida parapsilosis. After incubation for one or three weeks, the fragments were collected and histological analysis was performed. Results obtained in untreated nails evidenced, as expected, that the fungal invasion was different depending on the species: it was completed with Trichophyton spp., partial with Scopurlariopsis sp. and limited to the surface with Candida sp.. On the other hand, HPCH-treated nails were not invaded by fungal elements, neither dermatophytes nor moulds or yeasts. Besides showing the barrier effect of HPCH this paper describes a novel in vitro model of nail infection that is simple, reproducible and closely represents the in vivo human situation.
\end{abstract}

Keywords: Dermatophytes, hydroxypropyl chitosan, in vitro human nail model, moulds, onychomycosis, prevention, yeasts.

\section{INTRODUCTION}

Onychomycosis is responsible for up to $40 \%$ of all nail disorders, most of which (90-95\%) are caused by dermatophytes, especially T. rubrum and T. mentagrophytes. Yeasts (Candida spp.) and non-dermatophytic moulds (Aspergillus spp., Acremonium spp., Fusarium spp. and Scopulariopsis brevicaulis) account for the rest of nail infections [1]. Dermatophytes are soil saprophytes that evolved as parasites of animal keratinous tissues. They invade the nail structure directly and deeply [2]. Conversely, yeast and non-dermatophyte moulds, which are devoid of keratinolytic enzymes, infect firstly the soft tissues around the nails and they reach the nail secondarily, infecting only its surface [2].

The impaired integrity of nail structure plays an essential role in onychomycosis. Several risk factors promote fungal colonisation and infections: nail trauma, aging, damage in the nail structure such as enlarged porosity and intercellular spaces. The preventive measures commonly suggested to reduce the risk of infection are the implementation of hygienic measures, the use of comfortable and perspiring shoes and, in some cases, the treatment with antimycotic agents at a reduced dosage. However, no clear evidence of efficacy was ever proved with any of these measures. In addition, the use of low-dose antifungal agents is at high risk of inducing resistances [3]. In the light of these considerations, any non-pharmacological measure capable of protecting the nail of subjects at high risk of onychomycosis is of considerable value.

A new medical device (Myfungar ${ }^{\circledR}$, Polichem S.A.), based on Hydroxypropyl Chitosan (HPCH), was developed

*Address correspondence to this author at the Scientific Department, Polichem S.A., via Senago 42D, 6912 Lugano Pazzallo, Switzerland; Tel: +41.91.986 40 54; Fax: +41.91.986 40 20; E-mail: anna.bulgheroni@polichem.com by Polichem S.A. HPCH, a semisynthetic water-soluble derivative of chitosan, is a film forming water-soluble biopolymer endowed with several medically and cosmetically useful properties. Among them its capability to form a protective and long lasting barrier, when applied on the nail surface, is of utmost importance in the prevention of onychomycosis [4-6].

Procedures to test the efficacy of ready-to-use formulations have been set up but they are not devoid of limitations such as the absence of a nail substrate or of keratin [7].

In these regards, a fungal infection model based on human nails was set up. The experimental conditions, to monitor the fungal invasion of human nails in the presence or absence of the barrier formed by the HPCH-based medical device, were developed and tested with dermatophytes, yeasts and moulds responsible for onychomycosis.

\section{MATERIALS AND METHODS}

\section{Test Item}

Nail solution (Myfungar ${ }^{\circledR}$, Polichem S.A.) containing 1\% hydroxypropyl chitosan, and preservatives in a hydroalcoholic solution.

\section{Fungal Strains}

The species selected for these experiments represent the main causative agents of onychomycosis [5]: reference strains representative of dermatophytes, moulds and yeasts were obtained from the Deutsche Sammlung von Mikroorganismen und Zellkulturen GmbH (German Collection of Microorganisms and Cell Cultures: T. rubrum DSM 4167, T. mentagrophytes DSM 4870, C. parapsilosis DSM 11224 and 
S. brevicaulis DSM 9122). Strains were grown on Sabouraud Dextrose Agar (SDA) at $35 \pm 1{ }^{\circ} \mathrm{C}$.

\section{Preparation of Human Fingernail Fragments}

Distal fingernail clippings $(0.9 \mathrm{~cm}$ minimum $)$ were obtained from four healthy adult volunteers. Inclusion criteria were: no use of nail varnish or polish and no signs of fingernail disease for at least six months. Subjects were required to cut and collect the distal free edge of their fingernails using scissors or standard nail clippers. Cut nail fragments were soaked and shaken for $1 \mathrm{~h}$ in $70 \%$ ethanol, removed, dried and finally stored in sterile vials until the time of the experiment.
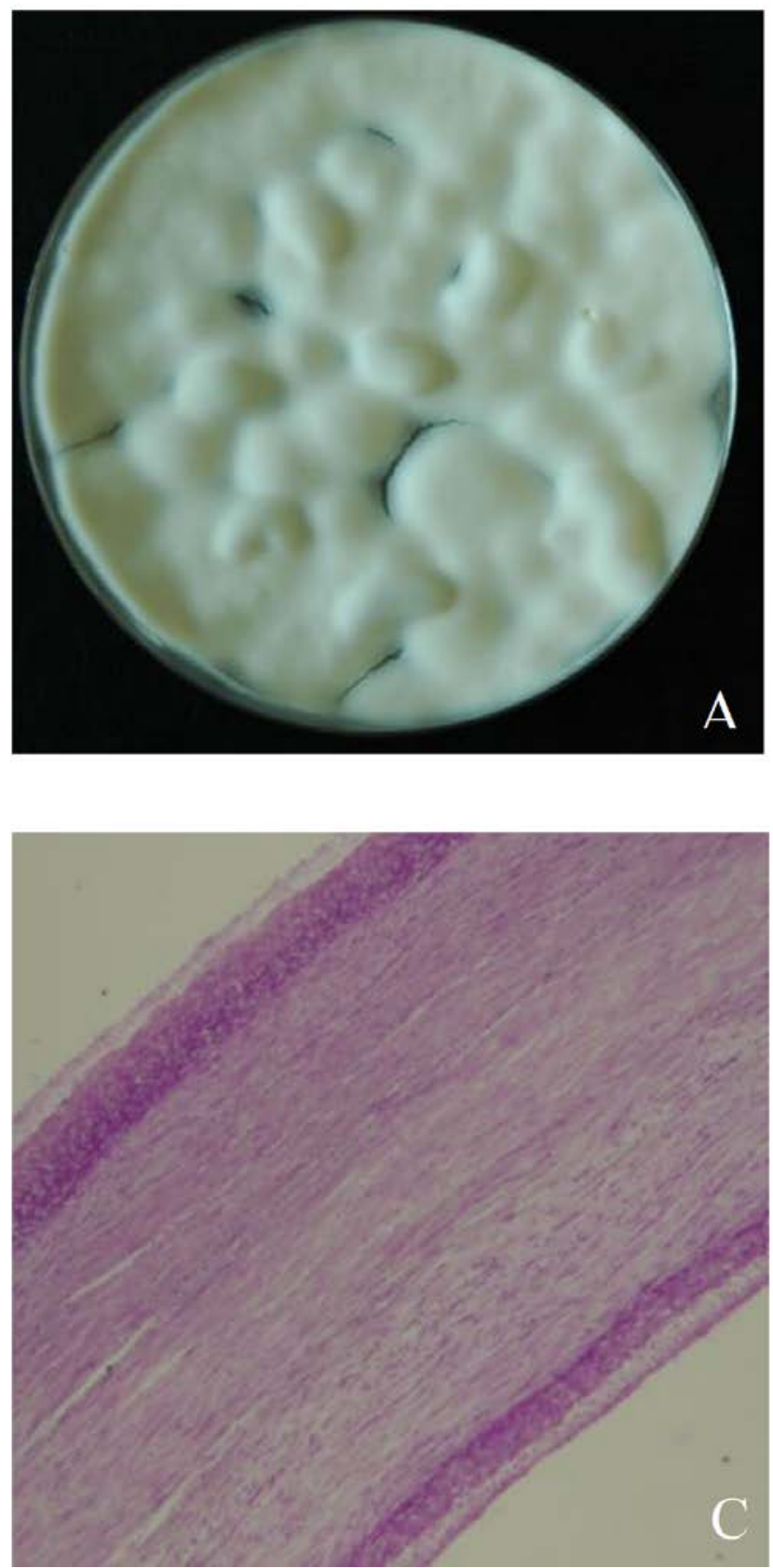

\section{Experimental Procedures}

The aim of this experiment was to evaluate the protective effects of an $\mathrm{HPCH}$ on human nails against fungal infections. SDA plates were inoculated with fungi (T. rubrum, $T$. mentagrophytes, $S$. brevicaulis or $C$. parapsilosis) and incubated until a well defined fungal growth was visible all over the plate surface. The size of the fragments was approximately $0.9-1.5 \mathrm{~cm}$ in length and $350-500 \mu \mathrm{m}$ thick. A volume of $20 \mu \mathrm{l}$ of the test product (HPCH-based medical device) was applied as a thin layer on the nail fragments by means of a brush and let dry. Controls were left untreated. Treated and untreated fragments were subsequently placed on the surface of the plates inoculated with the fungi and incubated for 7 or 21 days. At day 7 and day 21, nails were
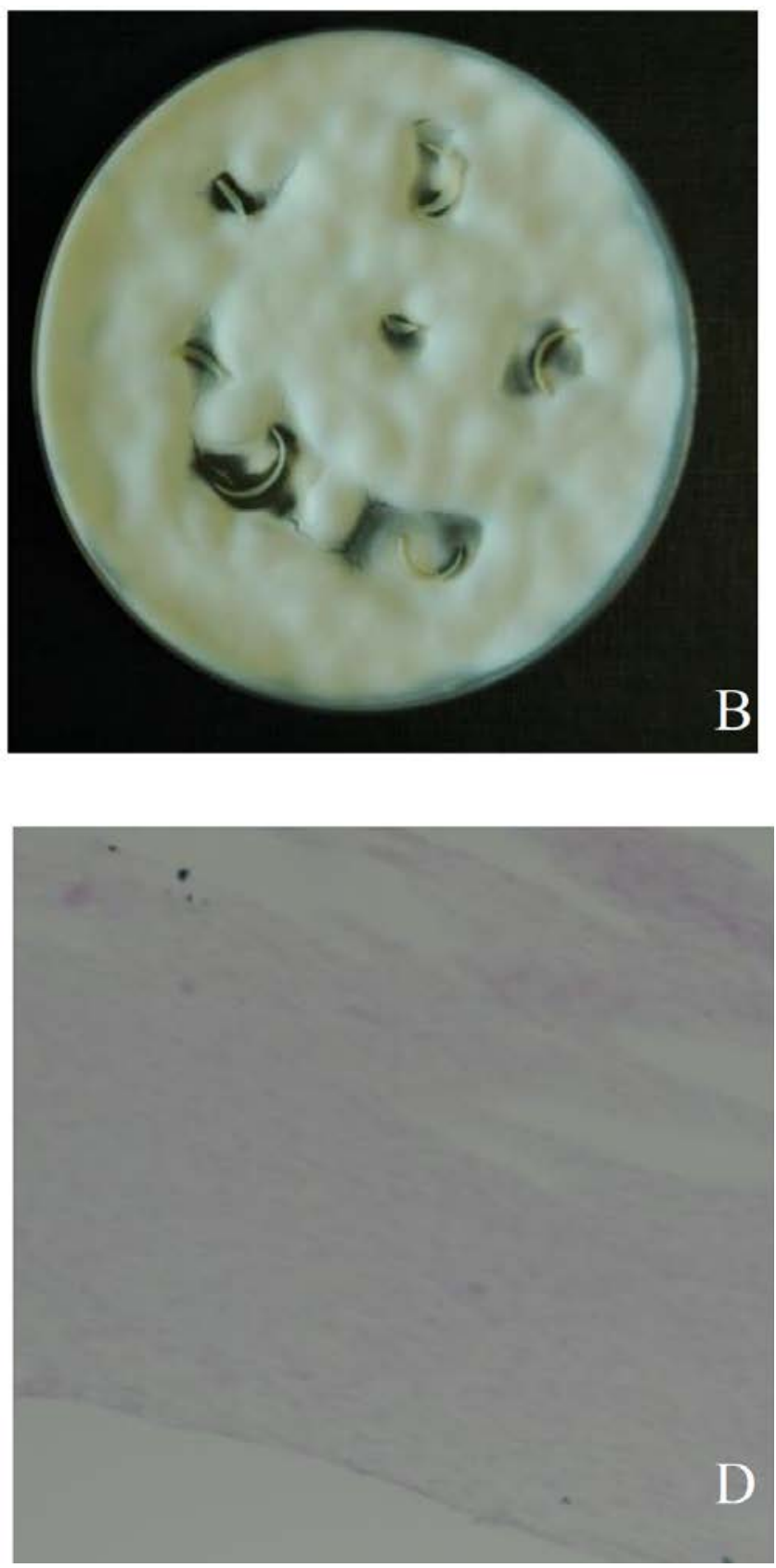

Fig. (1). Trichophyton rubrum following 7 days of incubation. Fungal growth on untreated (A) and treated nails (B). Fungal invasion of the nails of untreated $(\mathbf{C})$ and treated $(\mathbf{D})$ nails (100X; PAS stained). 
removed from the culture and observed macroscopically and microscopically, following PAS (Periodic acid-Schiff) staining.

\section{RESULTS}

Results here reported are representative of 3 to 4 different experiments.

Dermatophytes - All untreated nails were completely surrounded by fungal mycelium when put in contact with $T$. rubrum and T. mentagrophytes (Fig. 1A: T. rubrum; Fig. 2A: T. mentagrophytes) already after 7 days of incubation; on the contrary, those treated with the HPCH solution remained free from fungal colonization (Fig. 1B: T. rubrum; Fig. 2B: $T$.
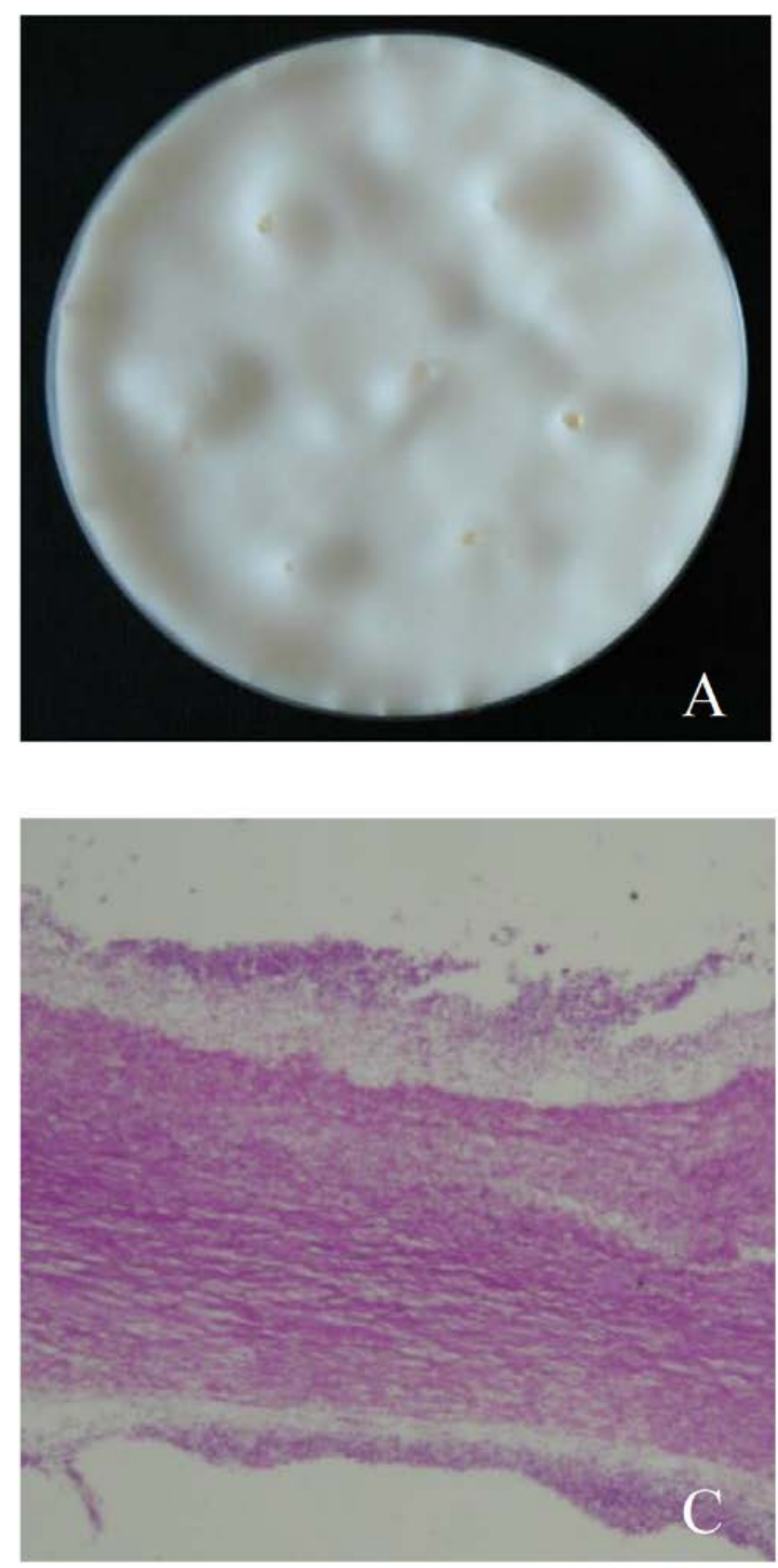

mentagrophytes). At PAS stain, both dermatophytes were able to invade the whole thickness of untreated nails already after 7 days (Fig. 1C: T. rubrum; Fig. 2C: $T$. mentagrophytes). Conversely in the HPCH treated nails only few fungal elements were visible at the external margins that had been in contact with the fungal culture (Fig. 1D: $T$. rubrum; Fig. 2D: T. mentagrophytes).

Moulds and yeasts - No macroscopically visible growth was observed on either untreated or $\mathrm{HPCH}$ treated nails, following incubation with $S$. brevicaulis or $C$. parapsilosis (Fig. 3A, B: S. brevicaulis; Fig. 4A, B: C. parapsilosis). As far as $S$. brevicaulis is concerned, in the untreated nails, PAS stain results were suggestive of a mild invasion of the nail, limited to the external margins. Contrary to the other test
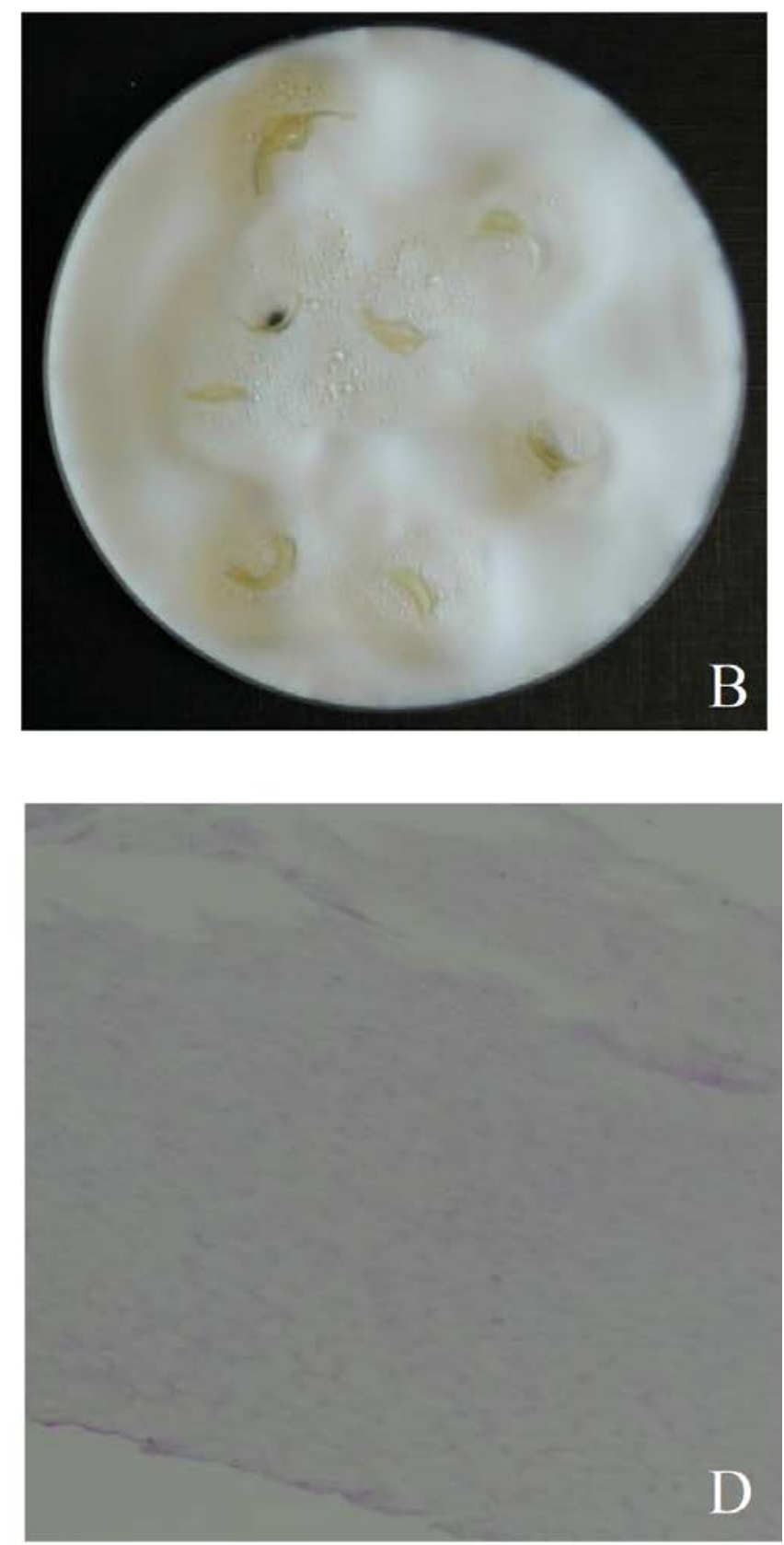

Fig. (2). Trichophyton mentagrophytes following 7 days of incubation. Fungal growth on untreated (A) and treated nails (B). Fungal invasion of the nails of untreated (C) and treated (D) nails (100X; PAS stained). 

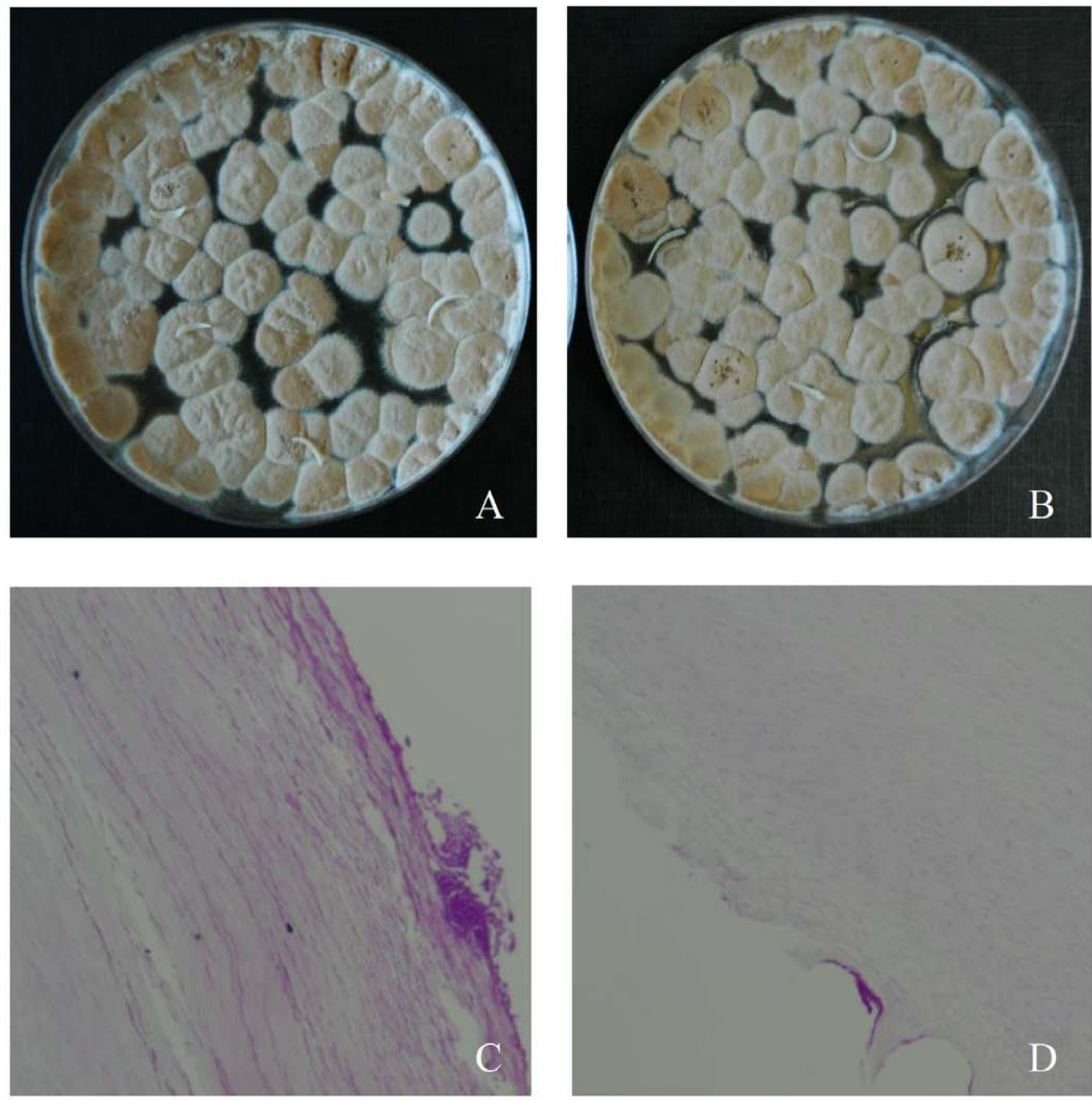

Fig. (3). Scopulariopsis brevicaulis, following 7 days of incubation. Fungal growth on untreated (A) and treated nails (B). Fungal invasion of the nails of untreated (C) and treated (D) nails (100X; PAS stained).

strains, fungal invasion progressed from day 7 to day 21 (Fig. 3C). No signs of colonisation or invasion were observed in the HPCH-treated nails (Fig. 3D).

Untreated nails incubated with C. parapsilosis showed an intense and uniform violet staining at PAS but limited to the surface of the preparation (Fig. 4C). PAS staining was totally absent in the HPCH treated samples (Fig. 4D).

\section{DISCUSSION}

It is increasingly being recognised that onychomycosis is a disorder that needs to be prevented and treated, providing impetus for the search of new models for the identification of novel antimycotic agents/formulations [2]. Procedures to test the efficacy of actives are available: the M27-A3 and M38A2 assays from Clinical and Laboratory Standards Institute [8] use drug solutions brought into a medium previously inoculated with suspended fungi or spores. However, these methods do not consider other factors involved in the development of the disease, e.g. the presence of keratin at the infected site. Keratin is not only a nutrient to the dermatophytes but it is also the up-regulator for some putative virulence factors [5]. The importance of keratin for mimicking the in vivo situation was the object of various studies [9-11], where the medium was supplemented with 

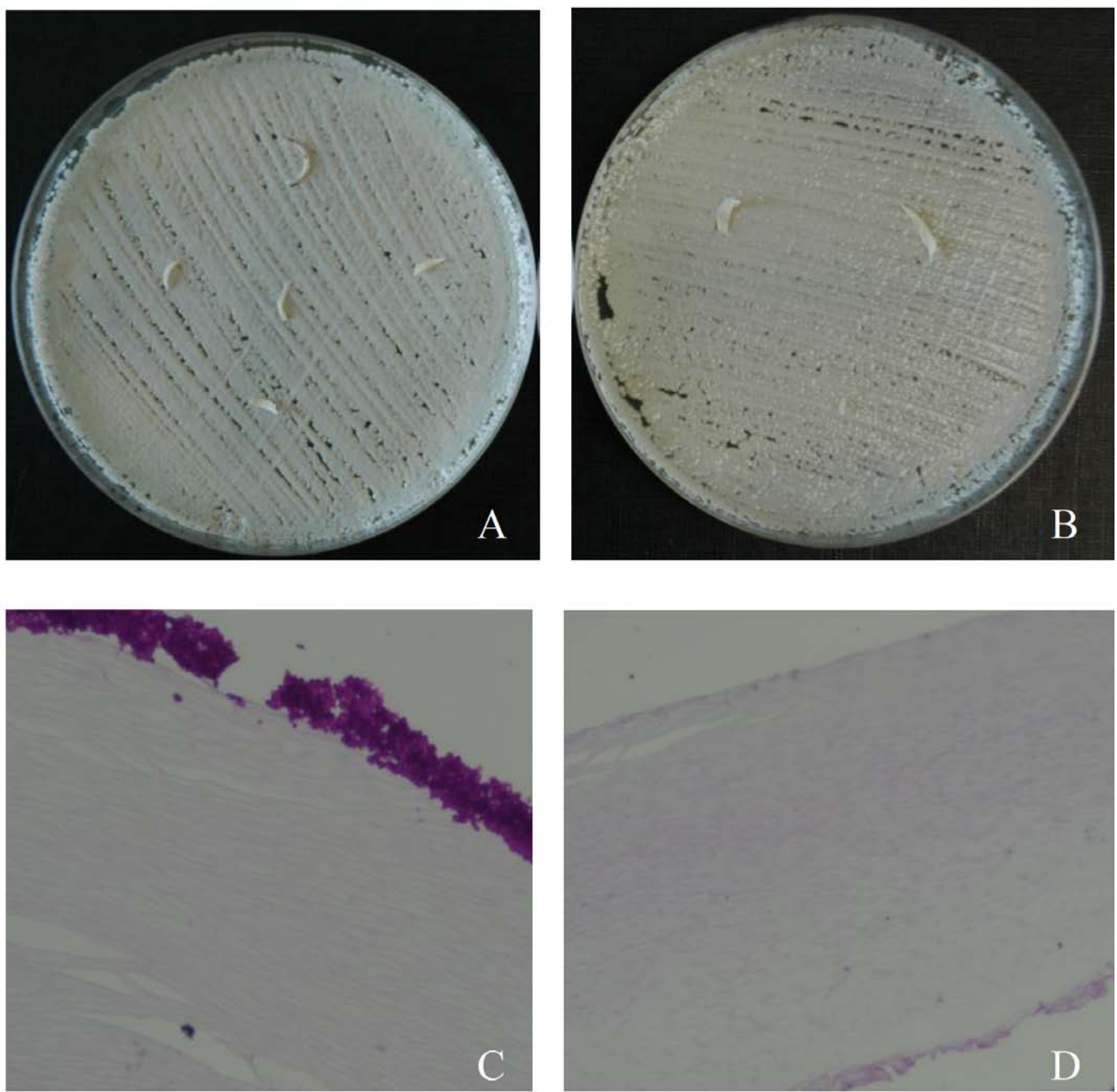

Fig. (4). Candida parapsilosis, following 7 days of incubation. Fungal growth on untreated (A) and treated nails (B). Fungal invasion of the nails of untreated $(\mathbf{C})$ and treated $(\mathbf{D})$ nails (100X; PAS stained).

pulverized nail clippings as the keratin source [5]. Lusiana et al. [5] reviewed the limitations of the current in vitro tests and proposed a novel model of infected nail plate using a keratin film made of human hair keratin, a largely available material, and compared it with bovine hoof, obtaining equivalent responses following treatment with antifungal preparations.

The method developed in this study uses intact human fingernail clippings and reflects more closely the natural physio-pathological conditions. This method proved simple, reproducible and representative of the in vivo human situation, although it was an in vitro study and as such it was not devoid of limitations. In particular, both sides of the nail were treated, thus not completely reflecting the actual in vivo treatment, but the treatment of one side would have resulted in the infection starting and progressing from the other side.

Our results in untreated nails are in good agreement with the known mechanisms of infection of the three tested fungal strains. The macroscopic and microscopic analyses of untreated nails showed that both dermatophytes were able to invade the whole thickness of the nail, while $S$. brevicaulis and $C$. parapsilosis remained limited to the surface. $T$. rubrum and $T$. mentagrophytes penetrated quickly into the nails and invaded them massively, the invasive process being completed within 7 days. S. brevicaulis invaded untreated nails rather slowly and only superficially. Analogously, $C$. 
parapsilosis did not invade untreated nails and proliferated only on the nail surface, similarly to what is known in humans [4].

This in vitro model was used to test the ability of the $\mathrm{HPCH}$-based medical device to prevent fungal infection of the nail. After a single treatment with the HPCH-based device, the nail fragments were protected from fungal colonization and this protection was long-lasting (over seven days). The results obtained in this study correlate with a recent publication in which HPCH treated bovine hoof slices were more resistant to penetration of dermatophytes with respect to untreated slices (e.g. penetration by $T$. mentagrophytes was $<25 \mu \mathrm{m}$ versus $275 \mu \mathrm{m}$ in the untreated control, at day 9) [12], while the application of commonly used aggressives, like isopropyl alcohol or urea, increased the ability of those pathogens to invade the nails. The results obtained in both studies can be attributed to the ability of the $\mathrm{HPCH}$ solution to form a barrier against the pathogens, as the product does not show any antifungal activity.

As previously reported the HPCH solution, applied to the surface of bovine hoof membranes, results, at scanning electron microscope, in smoother hooves, more resistant to mechanical insults thanks to the formation of a thin film [13].

These effects are of notable interest and indicate an additional potential beneficial role, in the prevention of onychomycosis in subjects at high risk or as a maintenance program following an efficacious antimycotic treatment, to avoid recurrences or reinfections, avoiding the risk to select resistant strains.

A recently published preliminary clinical trial reported significant benefits in the prophylaxis of onychomycosis in patients at risk [14]. Further studies may be needed to confirm those results.

\begin{tabular}{|c|c|c|}
\hline \multicolumn{3}{|c|}{ ABBREVIATIONS } \\
\hline$C$ & $=$ & Candida \\
\hline DSM & $=$ & $\begin{array}{l}\text { Deutsche Sammlung von Mikrorganismen } \\
\text { und Zellkulturen }\end{array}$ \\
\hline $\mathrm{HPCH}$ & $=$ & hydroxypropyl chitosan \\
\hline PAS & $=$ & Periodic acid-Schiff \\
\hline$S$. & $=$ & Scopulariopsis \\
\hline SDA & $=$ & Sabouraud Dextrose Agar \\
\hline sp. & $=$ & species singular \\
\hline spp & $=$ & species pluralis (multiple species) \\
\hline$T$ & $=$ & Trychopyton \\
\hline
\end{tabular}

\section{CONFLICT OF INTEREST}

The present work was funded by Polichem S.A. Authors are either employees (A.B. and L.F.) or former employee and/or consultant (F.M. and A.S.) of Polichem S.A.

\section{ACKNOWLEDGEMENTS}

We gratefully acknowledge the contribution of Giuseppe Togni and colleagues of IPAS laboratory (Ligornetto, Switzerland) for performing the microbiological work.

\section{REFERENCES}

[1] Murdan S. Topical nail products and ungual drug delivery. Boca Raton (USA), CRC Press 2013; pp. 1-35.

[2] Elewski BE. Onychomycosis: Pathogenesis, Diagnosis, and Management. Clin Microbiol Rev 1998; 11: 415-29.

[3] Daniel CR 3rd, Jellinek NJ. Commentary: the illusory tinea unguium cure. J Am Acad Dermatol 2010; 62: 415-7.

[4] Niewerth M, Korting HC. Candida albicans and the principle of opportunism: an assay. Mycoses 2002; 45: 253-8.

[5] Lusiana RS, Müller-Goymann CC. Infected nail plate model made of human hair keratin for evaluating the efficacy of different topical antifungal formulations against Trichophyton rubrum in vitro. Eur J Pharm Biopharm 2013; 84: 599-605.

[6] Tosti A, Hay R, Arenas-Guzmán R. Patients at risk of onychomycosis- risk factor identification and active prevention. J Eur Acad Dermatol Venereol 2005; 19 Suppl 1:13-6.

[7] Monti D, Saccomani L, Chetoni P, Burgalassi S, Saettone MF, Mailland F. In vitro transungual permeation of ciclopirox from a hydroxypropyl chitosan-based, water soluble nail lacquer. Drug Dev Ind Pharm 2005; 1: 11-7.

[8] National Committee for Clinical Laboratory Standards. Reference method for broth dilution antifungal susceptibility testing of conidium-forming filamentous fungi. Approved standard M38-A. Wayne, PA: NCCLS 2000.

[9] Osborne CS, Leitner I, Favre, Ryder NS. Antifungal drug response in an in vitro model of dermatophyte nail infection. Med Mycol 2004; 42: 159-63.

[10] Nowrozi H, Nazeri G, Adimi P, Bashashati M, Emami M. Comparison of the activities of four antifungal agents in an in vitro model of dermatophyte nail infection. Indian J Dermatol 2008; 53 125-8.

[11] Schaller M, Borelli C, Berger U, et al. Susceptibility testing of amorolfine, bifonazole and ciclopiroxolamine against Trichophyton rubrum in an in vitro model of dermatophyte nail infection. Med Mycol 2009; 47: 753-8.

[12] Ghannoum MA, Long L, Isham N, et al. Ability of Hydroxypropyl Chitosan nail lacquer to protect against dermatophyte nail infection. AAC 2015; 59: 1844-8.

[13] Sparavigna A, Setaro M, Frisenda L. Physical and microbiological properties of a new nail protective medical device. J Plastic Dermatol 2008; 4: 5-12.

[14] Chimenti S, Difonzo E, Aste N, Frisenda L, Caserini M. The protective efficacy of a new Hydroxypropyl Chitosan -based product in subjects at risk of onychomycosis. J Plastic Dermatol 2013; 9: 185-9. 